\title{
INEQUALITIES FOR THE INCOMPLETE GAMMA FUNCTION
}

\author{
Pierpaolo Natalini AND Biagio Palumbo
}

Abstract. We prove some monotonicity results for the incomplete gamma function,

$$
\Gamma(a, x)=\int_{x}^{\infty} e^{-t} t^{a-1} d t,
$$

from which some inequalities for $\Gamma(a, x)$ follow.

Mathematics subject classification (1991): 33B15.

Key words and phrases: Inequalities, Asymptotic formulas, Incomplete Gamma function.

\section{REFERENCES}

[1] M. Abramowitz And I. A. Stegun, EDS., Handbook of Mathematical Functions with Formulas, Graphs and Mathematical Tables, Dover, New York, 1965.

[2] A. LAFORGIA AND S. SiSMONDI, Monotonicity results and inequalities for the gamma and error functions, J. Comp. Appl. Math. 23 (1988), 25-33.

[3] A. LAFORGIA AND A. ElBERT, An inequality for the product of two integrals relating to the incomplete Gamma function (to appear) on J. In. Appl.

[4] F. QI AND S.L. GUO, Inequalities for the incomplete gamma and related functions, Math. Inequal. Appl. 2 (1999), 47-53. 